\title{
Mit 66.000 Betten um die Welt
}

\author{
Die Linet Group - alias Wissner-Bosserhoff - schreckt vor sperrigen Märkten in Über- \\ see und Asien nicht zurück. Ihre Abenteuerlust bewährt sich: Seit Jahren verzeichnet der \\ Bettenhersteller aus Wickede an der Ruhr ein jährliches Umsatzplus von zehn Prozent.
}

A n deutschen Krankenhäusern verzweifeln zurzeit die Medizintechnikfirmen. Den Kliniken fehlt das Geld für Investitionen, sie schieben Neuanschaffungen hinaus, bestellen vor allem Ersatzteile. In dieser Situation geht manchem Unternehmen die Geduld aus. Nicht aber der Linet Group, hierzulande besser bekannt unter dem Namen Wissner-Bosserhoff. Die deutschen Krankenhäuser behalten ihre Betten oft 20 bis 30 Jahre - trotzdem bemüht sich der größte europäische Hersteller von Krankenhaus- und Pflegebetten fast liebevoll um sie: So nimmt der Linet-Vorstand und Wissner-Bosserhoff-Geschäftsführer Michael Rosada die Eigenart einiger deutschen Kliniken, ihre Betten in einer Waschstraße zu reinigen, sehr ernst. „Hygiene ist ein wichtiges Thema im Klinikalltag, das auch die Betten betrifft. Aber wir kümmern uns darum, indem wir Produkte entwickeln, die besonders gut waschbar sind.“ Die
Kundenperspektive ist das, was für die Linet Group ganz offenbar zählt. Von Arroganz und Herablassung ist keine Spur, wenn Rosada, der viele Jahre für Marketing-Beratungen gearbeitet hat, über die deutschen Kunden spricht. Auf

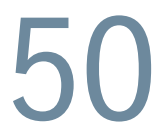

Mitarbeiter kümmern sich um den Vertrieb in den USA (North Carolina), wo die Linet Group SE nach eigener Aussage „ein hohes zweistelliges Umsatzwachstum“ erzielt.

die Frage, warum die Linet Group zurzeit so viele Anzeigen schaltet, antwortet er: „Man soll sich nicht vormachen, dass alle einen kennen, nur weil man schon viele Jahre im Markt ist. Es gibt immer wieder Leute, die sagen: ,Klar, Wissner-Bosserhoff kennen wir - das sind die mit den Papierhandtüchern, nicht wahr?' Wir müssen unseren Kunden schon sagen, was wir ihnen anbieten können und wofür wir stehen."

\section{Auf US-Markt sonst nur Konzerne}

Es gibt Länder auf dieser Welt, in denen muss die Linet Group bald nicht mehr viel erklären. In Brasilien etwa hat der Bettenhersteller eine Ausschreibung des Erziehungsministeriums gewonnen und stattet 46 Ausbildungskliniken mit mehr als 8.000 HightechKrankenhausbetten für die Universalund Intensivpflege aus. Seit 2006 hat er rund 16.000 Stück verkauft. „Das Gesundheitssystem in Brasilien wird ausgebaut. Auf diesen Wachstumsmarkt können wir uns noch besser einstellen, seit wir dort vor zwei Jahren Linet do Brasil gegründet haben“, sagt Geschäftsführer Rosada.

In bislang 13 Ländern ist die Linet Group mit eigenen Vertriebstöchtern 


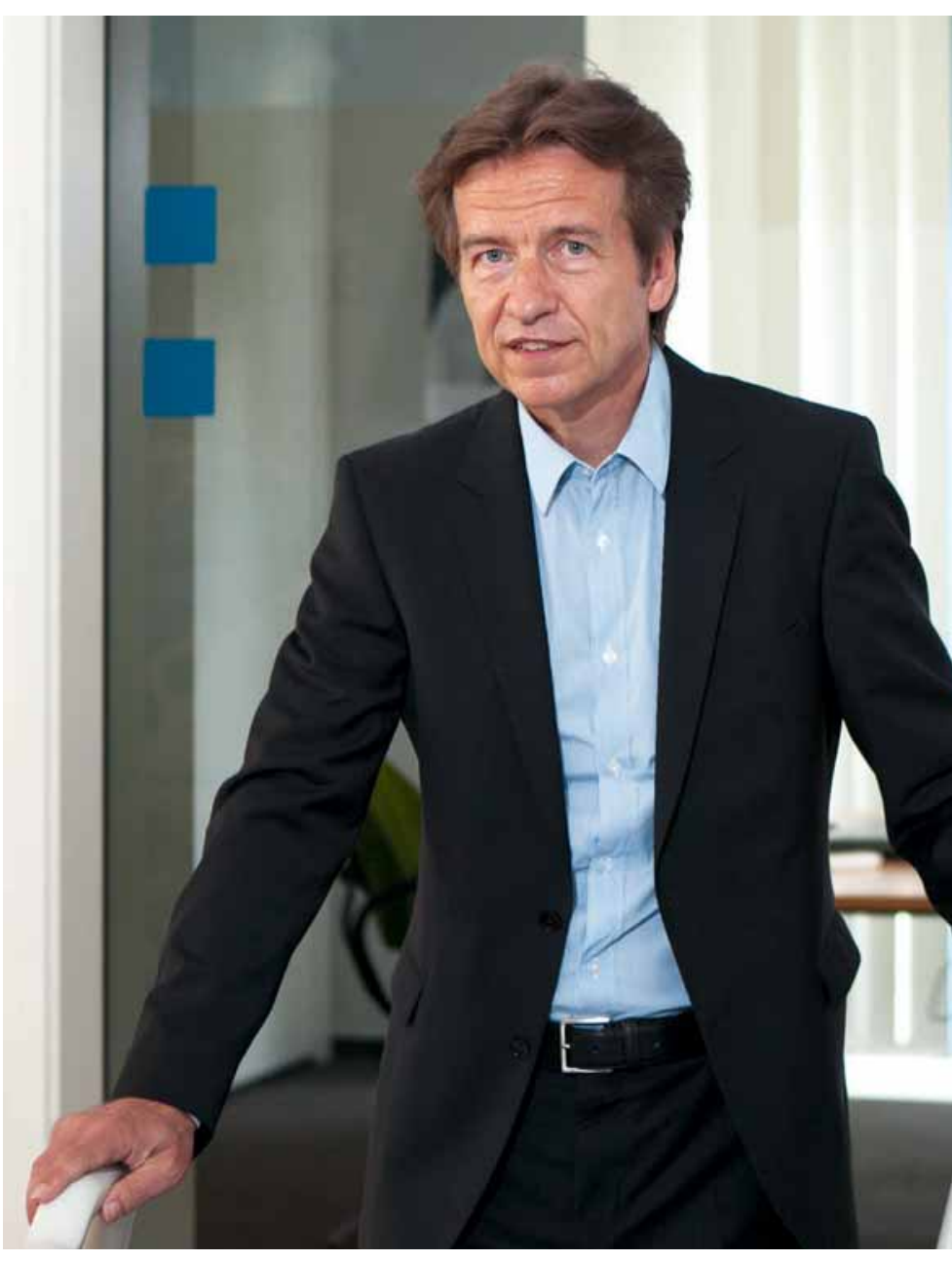

präsent. Auch in den USA hat der Bettenhersteller in jüngster Zeit Fuß gefasst, was für ein Unternehmen mit einem Umsatz von rund 147 Millionen Euro relativ ungewöhnlich ist. Normalerweise sind es große Medizintechnikkonzerne, die es auf den US-Markt zieht; Mittelständler konzentrieren sich auf die klassischen Wachstumsmärkte wie Lateinamerika und Asien. „Unser Produktportfolio trifft den amerikanischen Geschmack“, meint Michael Rosada. „Unsere Betten sind leichter als amerikanische, sehr funktional und haben das wird besonders gern gesehen - einen Simplicity-Wins-Effekt: Sie sind von den Schwestern leichter zu handhaben und zu transportieren. Hinzu kommt: Preislich sind wir wettbewerbsfähig, weil wir in unserem Werk im tschechischen Slaný bei Prag günstig und effizient produzieren können.“

In den USA erzielt die Linet Group, seit sie den Standort Charlotte (North Carolina) aufgebaut hat, mit 50 Mitarbeitern „ein hohes zweistelliges Umsatzwachstum pro Jahr“. Und das bei einem sehr regulierten Markt für Medizinprodukte. „Man muss in Group Purchasing Organizations gelistet sein. Diese sogenannten GPOs vertreten um die 2.000 Krankenhäuser. Wenn man gelistet ist, hat man sehr gute Chancen zu verkaufen. Wir haben glücklicherweise für alle GPOs die Verträge unter Dach und Fach“, berichtet LinetVorstand Rosada.

\section{Zur Person}

Michael Rosada ist seit 2010 Vorstand der Linet Group, die in Deutschland besser unter dem Namen Wissner-Bosserhoff bekannt ist. Davor war er Geschäftsführer bei einer international tätigen Beratungsfirma. Weitere Stationen seiner Karriere: McKinsey, Mercer Management Consulting, Capgemini, Tengelmann, Gerresheimer Glas und die Terrific AG, die er selbst gegründet hat. Michael Rosada ist hier zu sehen mit dem Multicare - „dem Flagschiff“ von Linet, wie er sagt. Das Multicare bietet unter anderem die Möglichkeit, den Bettrahmen schräg zu stellen (Lateralisierung), was Lagerung und Pflege des Patienten sehr erleichtert.

\section{Linet Group SE in Zahlen*}

\section{Umsatz: 146,7 Millionen Euro}

Ebitda-Rentabilität: 15,4 Prozent

Eigenkapitalquote: 70 Prozent

Verkaufte elektromotorische Betten: 66.000

Mitarbeiter: 881

*Geschäftsjahr 2013/2014 


\section{Red Dot Award für das Niedrigbett} Dass die Betten von Wissner-Bosserhoff Holzelemente enthalten und auf der Standardstation eine gewisse Hotelatmosphäre verbreiten, mag den US-Amerikanern gefallen, ein entscheidendes Kaufargument ist es für sie nicht. Denn in den USA sehen Krankenhausbetten, die dort vor allem aus Kunststoff gefertigt werden, traditionell recht klinisch aus. In Deutschland aber hat die wohnliche Note dem Unternehmen kürzlich - neben dem Plus X - den unter Architekten und Designern sehr bekannten Red Dot Award für das Niedrigbett Sentida beschert. „Die Verkleidungen in warmen Naturtönen lassen die technischen Funktionen in den Hintergrund treten und schaffen Behaglichkeit" - so die

\section{„Wenn wir es auf den chinesischen Markt schaffen, schlägt das alles, was wir bisher auf die Beine gestellt haben." Michael Rosada}

Begründung. Eine pflegewissenschaftliche Jury hätte es sicherlich etwas anders gewichtet. Denn das bis auf 27 Zentimeter absenkbare Bett mit zweiteiligen und mehrstufig höhenverstellbaren Seitensicherungen bewahrt Patienten vor schweren Stürzen, ohne dass sich Krankenschwestern oder Pfleger mit dem Problem der Freiheitsberaubung konfrontiert sehen.

Die Sentida-Modelle, mit denen Wissner-Bosserhoff den Preis gewonnen hat,

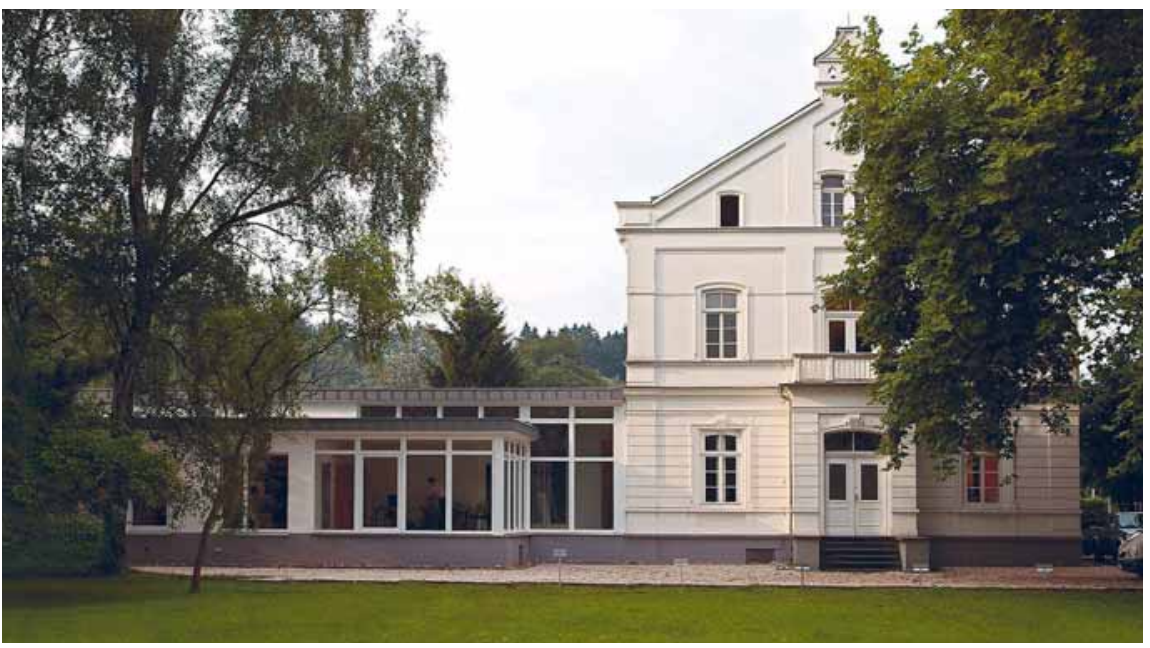

Der Firmensitz in Wickede: Hier ist Wissner-Bosserhoff 1952 gegründet worden. Heute sitzt in diesem Haus die Verwaltung inklusive der Geschäftsführung. In unmittelbarer Nachbarschaft werden die Pflegebetten gefertigt. Die Produktion von Klinikbetten der Linet Group findet in der Produktionsstätte in Slaný in der Nähe von Prag statt.

\section{Wissner-Bosserhoff oder Linet?}

In Deutschland ist der Bettenhersteller aus Wickede in Kliniken und Pflegeheimen gleichermaßen unter dem Namen WissnerBosserhoff bekannt. Doch im Ausland steht die Bezeichnung für den Geschäftszweig der Pflegebetten, Linet dagegen für das Klinikbetten-Geschäft. Wissner-Bosserhoff und Linet sind zugleich eigenständige Unternehmen, die seit 2011 unter dem Konzerndach der Linet Group SE agieren. stammen aus dem Nursing Home-Geschäftsbereich. „Allerdings gibt es ein speziell für den Krankenhausbereich konzipiertes Äquivalent, das Image 3 heißt und als ,2 in 1 Niedrigklinikbett' ausgelobt wird, da es neben der innovativen Sturzprävention auch alle herkömmlichen Elemente eines Krankenhausbetts beinhaltet", erzählt Rosada. Die beiden Geschäftsfelder Nursing Homes und Hospitals bereichern einander mehr und mehr: Auch der Nursing Home-Bereich integriert immer mehr Krankenhauselemente - etwa die eingebaute Waage, die sich auch als Bed-Exit-Assistent nutzen lässt. Sie sendet Impulse aus, wenn das angezeigte Gewicht plötzlich auf null zusteuert, weil der Patient das Bett verlässt.

\section{Matratzen, die Vitalzeichen messen} Die Bed-Exit-Lösung gehört natürlich zu den Dingen, die Kunden von einem modernen Klinikbettenhersteller erwarten. Das neueste Projekt aber ist eine echte Geschäftsfelderweiterung: Linet plant eine Matratze, die durch Sensoren die Vitalzeichen der Patienten kontrolliert. „Wir arbeiten in diesem Projekt mit Forschungsinstituten und einem Mathematikprofessor in Tschechien zusammen, der die Algorhythmen entwickelt. Zurzeit testen wir unsere Lösung im Prager Universitätsklinikum Motul“, erzählt Rosada. Das dürfte auch den Chinesen gefallen. Linet arbeitet seit zwei Jahren an einer Zulassung für den dortigen Markt. „Die Verfahren sind sehr langwierig, sehr bürokratisch. Wenn wir es aber schaffen und der Bedarf des Landes zum Tragen kommt, dann schlägt das alles, was wir bisher auf die Beine gestellt haben.“

Kirsten Gaede 


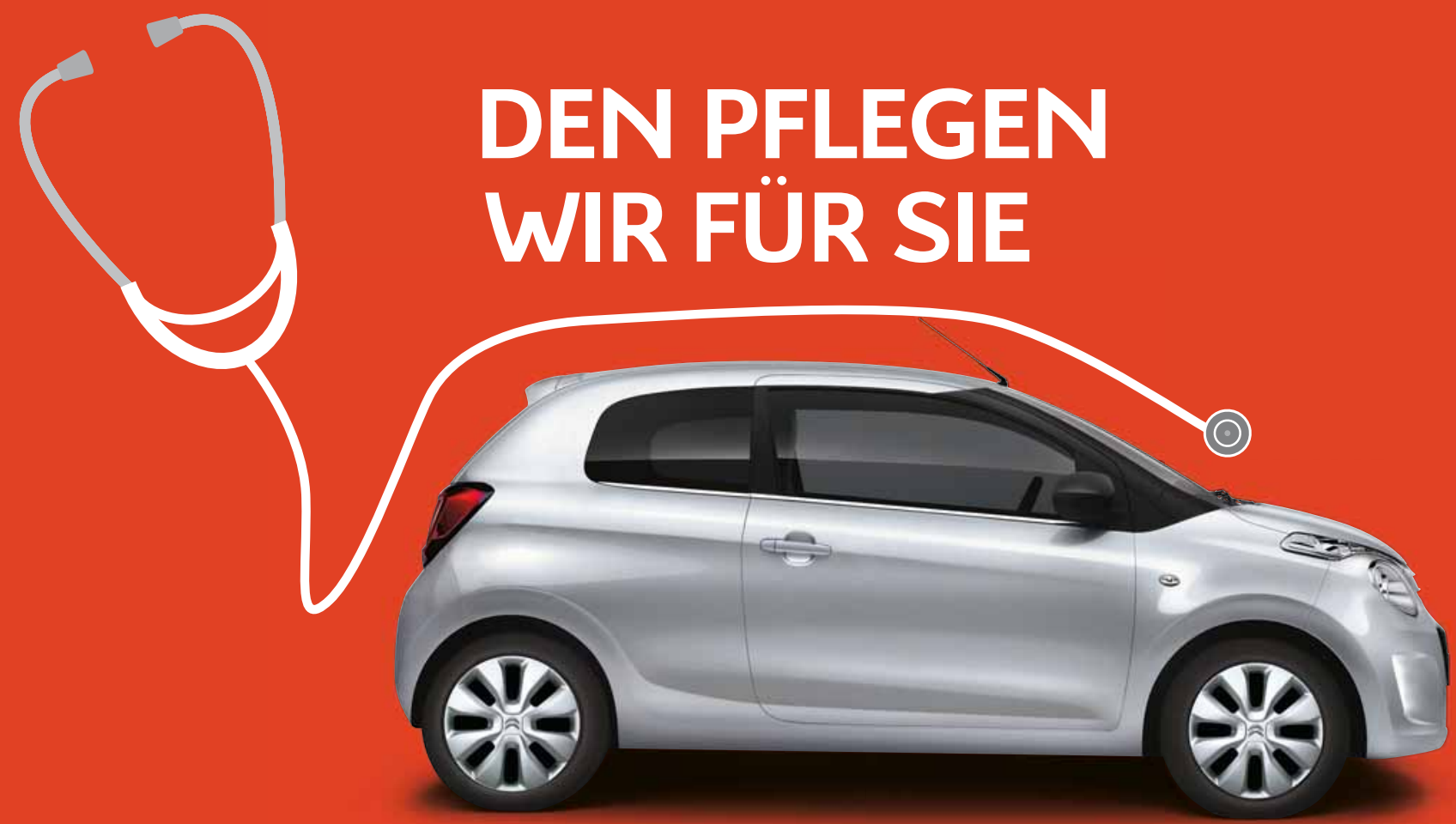

\section{CITROËN C1 VTi 68 Live}

$99 €_{m \rightarrow 1}^{\mathrm{mtl}}$

zzgl. MwSt. INKL. FULL SERVICE*

CITROËN empfiehlt TOTAL

DER NEUE CITROËN C1 MIT FULL SERVICE:

WARTUNG, GARANTIE UND ERSATZ VON VERSCHLEISSTEILEN.

Pflegeleichter geht's nicht: Der neue CITROËN C1 inklusive Wartung, Garantie und Ersatz von Verschleißteilen bleibt selbst im stressigsten Arbeitsalltag immer topfit. Und dank seines Audiosystems inklusive USB-Anschluss, der Klimaanlage und vielen weiteren Extras fehlt es auch Ihnen an nichts schon ab günstigen $99,-€$ im Monat.

*Ein Full Service Kilometerleasing-Angebot der Banque PSA Finance S.A. Niederlassung Deutschland, Geschäftsbereich CITROËN BANK, Siemensstraße 10, 63263 Neu-lsenburg, für Gewerbetreibende im Pflegedienst zzgl. MwSt. und Fracht für den CITROËN C1 VTi 68 LIVE, 3-Türer: Laufzeit 48 Monate, 10.000 km/Jahr Laufleistung, gültig bis zum 30.09. 2014. Leistungen gemäß den Bedingungen des CITROËN FreeDrive à la carte Vertrages der CITROËN DEUTSCHLAND GmbH, Edmund-Rumpler-Straße 4, 51149 Köln. Detaillierte Vertragskonditionen unter www.citroen.de. Nur bei teilnehmenden CITROËN Partnern. Abb. zeigt evtl. Sonderausstattung/höherwertige Ausstattung.

Kraftstoffverbrauch innerorts 5,0 l/100 km, außerorts 3,6 l/100 km, kombiniert 4,1 l/100 km, $\mathrm{CO}_{2}$-Emissionen kombiniert $95 \mathrm{~g} / \mathrm{km}$. Nach vorgeschriebenem Messverfahren in der gegenwärtig geltenden Fassung. Effizienzklasse: $B$. 